\title{
Comprehensive evaluation of botulinum toxin treatment response of a patient with Temporomandibular Disorder
}

\author{
Victoria Sitnikova ${ }^{1}$, Antti Kämppi ${ }^{2}$, Pentti Kemppainen ${ }^{3}$, and Olli Teronen ${ }^{1}$ \\ ${ }^{1}$ University of Helsinki Faculty of Medicine \\ ${ }^{2}$ Affiliation not available \\ ${ }^{3}$ Clinicum
}

February 2, 2021

\begin{abstract}
Botulinum toxin is widely used in the treatment of temporomandibular disorder symptoms even though consensus on this treatment modality has not yet been reached. Outcome assessment methods described herein could serve as a novel scientific method for the reliable evaluation of the effectiveness of botulinum toxin in further investigations.
\end{abstract}

\section{Introduction}

Temporomandibular disorders (TMDs) are neuromuscular and musculoskeletal disorders affecting the jaw and muscles of mastication, with pain being the most common complaint. Hyperactivity of masticatory muscles is a common background in TMD, which led to the hypothesis that muscle relaxation with botulinum toxin may be a treatment of choice. Botulinum toxin type A (BoNT-A) blocks the release of acetylcholine from the presynaptic membrane, leading to abridged muscle contraction. BoNT-A has also been suggested to have analgesic properties by blocking the release of substance $\mathrm{P}$, glutamate, and calcitonin gene-related peptide (CGRP) [1,2].

Abundant research has shown that BoNT-A injections reduce pain in patients with TMD, providing a new means for treating patients with refractory symptoms [3]. Nevertheless, some studies have reported no improvements of symptoms after treatment [4]. Contradictory results support the multifaceted nature of TMD. Since pain is the main symptom of TMD, most studies have concentrated on the pain-alleviating effect of BoNT-A, and clinical studies applying objective measurable parameters, such as electromyography (EMG), are missing [5]. Accordingly, there is an urgent need for controlled studies that assess subjective complaints and compare these with objective physical findings and provide a long-term follow-up (4-6 months). The TMD treatment response evaluation method presented in this case, including EMG and bite force measurements, provides a good tool for proper evaluation of treatment outcomes, taking into account the variable etiology and manifestation of TMD.

\section{Case history and examination}

The patient is a 53-year-old woman who's symptoms had lasted for over 30 years and the pain was troublesome 1-3 times per month. She sought treatment for facial pain and headache and self-reported bruxism. The patient had no systemic diseases, no medication and did not smoke. She had self indentified that symptoms correlatated often with stress episodes. The patient was treated with many different TMD treatment modalities such as splint therapy, non-steroidal anti-inflammatory drugs medication and soft food diet, but with poor success. 
The clinical examination revealed a maximum mandibular opening of $50 \mathrm{~mm}$ and a normal range of eccentric movements. No joint pain or sound was noted. There was a stable occlusal condition in a musculoskeletal stable position. According to the pain drawing, the pain that the patient experienced during the last six months was intraorally and in the areas of the head, neck, face, and shoulders throughout. Palpation of the muscles of mastication revealed muscle tenderness in the areas of the masseter and temporalis with no referred pain. Palpation-induced pain was familiar to the patient. The diagnosis was local myalgia and headache attributed to TMD secondary to parafunctional activity and stress-related muscle co-contraction. Since the symptoms were lasting for years and the conservative treatments of muscle pain were ineffective, activity within central nervous system (CNS) could be involved in the pain input. The diagnosis of centrally mediated myalgia could not be excluded. In this investigation we decided to try botulinum toxin injections as a treatment option and combined with comprehensive assessments of the treatment responses.

The Characteristic Pain Intensity (CPI) score was calculated from the 0-10 ratings of current, worst, and average pain intensity during the past six months. Before the treatment the score was 7.3. The chronic pain grade (CPG, baseline to six months, grades 0-IV) was II, corresponding to 'High intensity pain, without disability'. Jaw functional limitations were observed in the following activities: chewing tough food, smiling, yawning, and tooth brushing. The degree of pain-related worry was 7 (0-10 scale). Based on the Symptoms Checklist-90 Revised (SCL-90R) questionnaire, the patient had only mild symptoms of depression, with an average score of 0.5 (0-4 scale). The same questionnaire revealed severe sleep disorder, with an average score of 3.0 (0-4 scale). The average score of non-specific physical symptoms with pain items was 0.6 and non-specific physical symptoms without pain items 0.4 (0-4 scale). The initial average electromyography (AEMG) value of the masseter muscle during clenching with maximum force was $241 \mathrm{mV}$ and at rest 45 $\mathrm{mV}$, and the average maximum bite force was $580 \mathrm{~N}$ (Figure 1).

Clinical diagnosis was based on the standardized clinical examination protocol and Axis I decision tree of the Diagnostic Criteria for Temporomandibular Disorders (DC/TMD) [6]. Axis II biopsychosocial assessment instruments of the Research Diagnostic Criteria for Temporomandibular Disorders (RDC/TMD) were used to evaluate the effect of treatment on disability and psychological status [7]. The following questionnaires were used before the treatment and at each follow-up visit:

Axis I (Physical findings)

Demographics

Clinical examination form (DC/TMD)

Axis II (Disability \& Psychological Status)

- Pain Drawing

- None: number of body areas with pain $=0$

- Mild: number of body areas with pain $=1$

- Moderate: number of body areas with pain $=2$

- Severe: number of body areas with pain being over 3

- Graded Chronic Pain Scale (GCPS) 1.0

- 0: no TMD pain in the preceding six months

- I: low disability, low pain intensity

- II: low disability, high pain intensity

- III: high disability, moderately limiting

- IV: high disability, severely limiting

GCPS 1.0 grades are derived from seven questions measuring

- Characteristic pain intensity (CPI) (current, worst, average) (range 0-10;0 = no pain, $10=$ worst pain)

- Disability days 
- Pain-related interference with daily, social, and work-related activities

- Questionnaire for measuring jaw functional limitation (11 items withyes or no option: chewing, drinking, exercising, eating hard foods, eating soft foods, smiling/laughing, sexual activity, cleaning teeth or face, yawning, talking, having your usual facial appearance)

- Pain-related worry (range $0-10 ; 0=$ not at all worried, $10=$ extremely worried)

- Ability to control pain (range 0-6) or the ability to decrease pain (range 0-6)

- RDC/TMD_FIN Depression and Somatization Scores Based on the Symptom Checklist 90, Revised (SCL-90R) (5-point Likert scale: $0=$ not at all, $4=$ very much) including the following assessments:

- Symptoms of depression (20 questions)

- Sleep dysfunction (3 questions)

- Non-specific physical symptoms (including pain items) (12 questions)

- Non-specific physical symptoms (without pain items) (7 questions)

EMG of masseter muscle and bite forces were assessed before the treatment and after the botulinum toxin injections at each follow-up visit. A series of recordings consisted of an exercise lasting 60 seconds so that every 10th second the patient was asked to bite hard for 5 seconds followed by a 5 -second relaxation. The values of AEMG were selected from the middle of each 5-second relaxation and clench periods. Maximum bite force was selected from each clench period. Six values of AEMG and bite force were selected at each patient's visit, and the average values were calculated.

The patient received a one-time treatment of 50 units of BoNT-A (Xeomin®), supplied by Merz Pharmaceuticals GmbH, Germany); 2/3 of the dose was injected into the bilateral masseter muscles (16.7 units each masseter) and $1 / 3$ into the bilateral temporalis ( 8.3 units each temporalis). Three injections were applied along the inferior border of the masseter and two injections along the anterior part of the temporalis. Follow-ups were performed at 2, 11, 16, and 22 weeks after the botulinum toxin injections.

At two weeks after the injections, there were no significant changes in pain location, pain intensity, or degree of disability, with chronic pain grade remaining at II and CPI 6.7. However, functional limitations were no longer observed. The score for pain-related worry level was 6 (range 0-10). The following scores were obtained from the Axis II biobehavioural questionnaire (range 0-4): symptoms of depression 0.45, sleep dysfunction 1.7, and non-specific physical symptoms with and without pain items 0.3. The AEMG value during clenching with maximum force dropped to $55 \mathrm{mV}$ and the average maximum bite force to $305 \mathrm{~N}$ (Figure 1). No changes occurred to AEMG rest $(45 \mathrm{mV})$.

At 11 weeks after the injections, only the parietal region remained as a pain area. The chronic pain grade was 0 , meaning that TMD pain and its related disability were no longer present. No functional limitations were observed. Pain-related worry level was 1 (range 0-10). The following scores were obtained from the Axis II biobehavioural questionnaire (range 0-4): symptoms of depression 0.35, sleep dysfunction 1.0, and non-specific physical symptoms with pain items 0.2 and without pain items 0 . The AEMG value during clenching with maximum force was $74 \mathrm{mV}$, and the average maximum bite force was $326 \mathrm{~N}$ (Figure 1).

At 16 weeks after the injections, the pain distribution level was moderate with two areas affected: the temporalis and the right side of the neck. The severity of chronic pain was graded as II and CPI was 6.0. Nonetheless, no functional limitations were observed. Pain-related worry level was 5 (range 0-10). The following scores were obtained from the Axis II biobehavioural questionnaire (range 0-4): symptoms of depression 0.3, sleep dysfunction 1.7, and non-specific physical symptoms with pain items 0.3 and without pain items 0.1. The AEMG value during clenching with maximum force was $98 \mathrm{mV}$, and the average maximum bite force was $488 \mathrm{~N}$ (Figure 1).

At 22 weeks after the injections, the pain distribution level was still moderate, affecting the parietal and temporal regions. CPI score was 0, with no functional limitations. Pain-related worry level was 5 (range 0-10). The following scores were obtained from the Axis II biobehavioural questionnaire (range 0-4): symptoms of depression 0.25 , sleep dysfunction 1.0, and non-specific physical symptoms with pain items 0.2 and without 
pain items 0 . The AEMG value during clenching with maximum force was $133 \mathrm{mV}$, and the average maximum bite force was $452 \mathrm{~N}$, indicating that the physiological effect of BoNT-A was still present (Figure 1).

Ethics Approval

The study adhered to the tenets of the Declaration of Helsinki, and the regional Ethics Review Board of Helsinki University Hospital approved the study protocol (ID: HUS/3167/2018).

\section{Discussion}

Several studies have shown the efficacy of BoNT-A in treatment of TMD [3,8]. Different types and doses of botulinum toxin used in researches complicate the comparison of conclusions, as do variations in the outcomes measured, the timing and duration of follow-ups and the complexity of the diagnostics and etiologic factors of TMD. In this study, a beneficial effect was achieved with $50 \mathrm{U}$ of BoNT-A. Pain assessment tools revealed that the TMD symptoms of a 53-year-old woman were relieved for at least 22 weeks, with the best therapeutic gain achieved at 11 weeks. The reduction in TMD pain level correlated with the decrease in sleep dysfunction level and the improvement in jaw function. Pain-related worry decreased and some improvements in psychological factors were observed. The increased level of muscle activity as an etiologic factor in this case might support the beneficial effect of the botulinum toxin treatment since muscle relaxation was achieved. The fact that the pain was bothering the patient for years and CNS was exposed to prolonged nociceptive input, the beneficial effect might be explained by the botulinum toxin's effects on nociceptive neurotransmitters. In future investigations, whether botulinum toxin is effective in preventing pain to progress into a chronic pain condition or whether it is a treatment of choice in chronic pain problem yet should be studied.

The reduced EMG values of the masseter muscle after BoNT-A injections clearly show the physiologic effect of the drug. Before the injections, the AEMG was $241 \mathrm{mV}$ and two weeks later the value had dropped to $55 \mathrm{mV}$, which is only slightly higher than the AEMG value at rest $(45 \mathrm{mV}$ ) (Figure 1). A considerable reduction in muscle activity was observed throughout the study, i.e. up to 22 weeks. Decreased bite forces were observed; the mean value obtained before treatment was $580 \mathrm{~N}$, and after the injections the lowest values were seen at 2 weeks $(305 \mathrm{~N})$ and 11 weeks $(326 \mathrm{~N})$ (Figure 1).

In this case the patient did not experience any adverse effects during 22 months. The temporary side effects reported in the literature are localized pain, difficulty chewing, and focal muscle weakness. [3]. The repeated injections of BoNT-A into the masseter muscle may decrease bone volume in the mandibular angle which emphasizes that a treatment plan should be done judiciously and side effects should be monitored when multiple injections are planned [9]. As with any other treatment modality careful evaluation of etiologic factors of the disorder should be done since only the treatment directed to the source of the pain can lead to a successful result. A re-evaluation of the etiology should take place before repeating the injections.

\section{Conclusion}

This work highlights the importance of many factors when evaluating the outcomes of TMD treatment. Consistent assessment reporting tools would allow appropriate comparison of results, facilitating the development of a consensus on the therapeutic benefit of botulinum toxin. Since TMD pain is not always eliminated with conservative treatment methods, such as physical therapy or occlusal splint, other treatment options must be devised. The diagnostic tools and treatment response evaluation methods described herein serves as a novel model to perform a scientifically sound examination when investigating the efficacy of botulinum toxin and other treatment modalities in TMD patients.

\section{Disclosure of Interest}

The authors do not have any financial relationships that might lead to a conflict of interest.

\section{Funding Statement}

The authors received no specific funding for this work.

\section{Acknowledgements}


Merz Pharmaceutical GmbH is acknowledged for providing the botulinum toxin used in this study.

\section{Authorship}

VS: contributed to manuscript writing, performed material preparation. AK: responsible for the construction of the manuscript. PK: contributed to the study conception and design, diagnosing and examination. OT: contributed to the study conception and design. All authors were involved in the editing and final approval of the manuscript.

\section{References}

1. Aoki KR. Evidence for antinociceptive activity of botulinum toxin type A in pain management. Headache 43(Suppl 1): S9-15, 2003

2. Meng J, Wang J, Lawrence G, Dolly JO. Synaptobrevin I mediates exocytosis of CGRP from sensory neurons and inhibition by botulinum toxins reflects their anti-nociceptive potential. J Cell Sci 120(16):1864-74, 2007

3. Thambar S, Kulkarni S, Amstrong S, Nikolarakos D. Botulinum toxin in the management of temporomandibular disorder: a systematic review. Br J Oral Maxillofac Surg. Mar 03, 2020 [Epub ahead of print]

4. Ernberg M, Hedenberg-Magnusson B, List T, Svensson P. Efficacy of botulinum toxin type A for treatment of persistent myofascial TMDpain: a randomized, controlled, double-blind multicenter study. Pain 152(9):1988-96, 2011

5. Suvinen TI, Malmberg J, Forster C, Kemppainen P. Postural and dynamic masseter and anterior temporalis muscle EMG repeatability in serial assessment. J Oral Rehabil. 36(11):814-20, 2009

6. Ohrbach R, editor. Diagnostic Criteria for Temporomandibular Disorders: Assessment Instruments. Version 15May2016. [Diagnostiset Kriteerit Purentaelimistön Kivuille ja Toimintahäiriöille (DC/TMDFIN): Tutkimusinstrumentit: Finnish Version 25May2016] Sipilä K, Suvinen T, Trans.www.rdctmdinternational.org (Accessed on July 1, 2020).

7. Suvinen T, Rantala M, Ahlberg J, Könönen M. RDCTMD_FIN. www.rdc-tmdinternational.org (Accessed on July 1, 2020).

8. Pihut M, Wisniewska G, Majewski P, Gronkiewicz K, S Majewski. Measurement of occlusal forces in the therapy of functional disorders with the use of botulinum toxin type A. J Physiol Pharmacol 60(Suppl 8): 113-6, 2009

9. Lee HJ, Kim SJ, Lee KJ, Yu HS, Baik HS. Repeated injections of botulinum toxin into the masseter muscle induce bony changes in human adults: A longitudinal study. Korean J Orthod. 2017;47(4):222228

\section{Figure Legend}

Figure 1 . Box plots of the average electromyography (EMG) and bite force values. The measurements were done before the BoNT-A injections into the masseter and temporalis muscles (blue) and 2 weeks (red), 11 weeks (grey), 16 weeks (yellow) and 22 weeks (green) after the injections. Each box plot shows median (solid line inside the box), mean (cross inside the box), 25th and 75 th percentiles. 


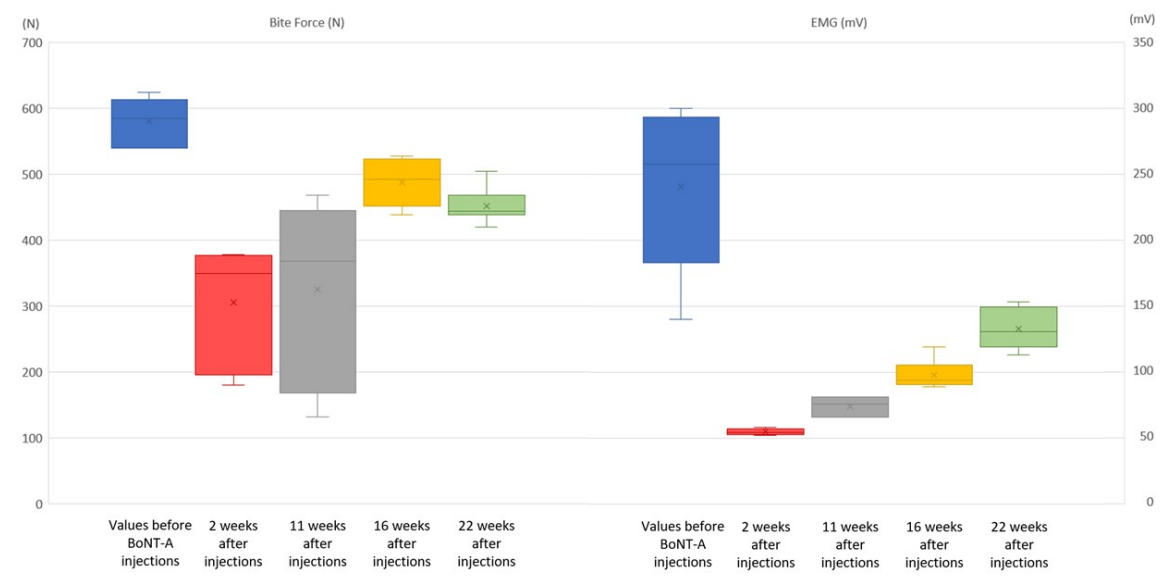

